Alla memoria di

Fernando Gascó

\title{
COMMODO E I SUOI APOLOGETI
}

The laudatory judgement on Commodus by the Christian poet Dracontius, contrary to the widespread historical tradition, springs from a favourable tradition diffused not only in the Christian sphere: the examination of contemporary inscriptions and of literary sources, such as Joannes Malalas and Eusebius, allows to reconstruct a wide favourable tradition, also in the pagan sphere, which praised Commodus' victories, exalted his reign as an age of universal peace and showed off, contrary to the senatorial tradition, the noteworthy advantages granted by the Emperor to the provinces. This tradition must have been very strong in Africa, where Dracontius lived, through the memory of Commodus' policy in favour of the African provinces.

Il poeta cristiano Draconzio, vissuto a Cartagine verso la fine del $\mathrm{v}$ secolo, ci ha lasciato in alcuni suoi versi una testimonianza di notevole interesse sulla fama dell'imperatore Comodo:
Alter ait princeps modico sermone poeta
Commodus Augustus, uir pietate bonus:
«Nobile praeceptum, rectores, discite post me:
sit bonus in uita qui uolet esse deus»!

Il riferimento di questi versi a Commodo ha suscitato perplessità, dato il contrasto fra il giudizio apertamente elogiativo sull'imperatore (uir pietate bonus) e l'atteggiamento nettamente ostile nei confronti di Commodo riscontrabile invece nella massima parte della tradizione storiografica, da Erodiano a Cassio Dione, ad Aurelio Vittore, ad Eutropio, fino ancora al cristiano Orosio $^{2}$. Non sono mancati, dunque, i tentativi di spiegare la testimonianza dra-

1 Dracont, satisf. $187-90$.

2 Sulla tradizione relativa a Commodo si vedano soprattutto F. Grosso, La lotta politica al tempo di Commodo, Mem. Accad. d. Sc. di Torino, Cl. di Sc. Mor., Stor. e Filol., Ser. IV, nr. 7, Torino 1964, p. 3 ss.; U. Espinosa Ruiz, "El reinado de Cómmodo: subjetividad y objetividad en la antigua historiografian, Gerión 2, 1984, pp. 113-49, con bibliografia. Sul racconto di Orosio (VII 16), che si basa su Girolamo ed Eutropio, esasperando la versione ostile di quest'ultimo, cf, anche Orosio. Le Storie contro i pagni, a cura di A. Lippold, II, Milano 1976, p. 486. 
conziana come un errore ${ }^{3}$; ma il riferimento di essa a Commodo è stato pure difeso ${ }^{4}$, ricollegandola ad una tradizione cristiana e facendo presenti da un lato la politica di Commodo favorevole ai cristiani, soprattutto per effetto dell'influenza della concubina Marcia, dall'altro le attestazioni, appunto in ambito cristiano, di giudizi positivi su Commodo ${ }^{5}$. Alcune delle testimonianze citate in proposito mi sembrano, tuttavia, poco pertinenti ${ }^{6}$, mentre altre attestazioni consentono, a mio avviso, di delineare in maniera più ampia e precisa il quadro di una tradizione favorevole a Commodo, non limitata al solo ambito cristiano, e di chiarime alcuni motivi e contenuti, a partire dai contemporanei dell'imperatore; esse valgono cosi, non solo a spiegare la testimonianza draconziana, ma anche a mettere in luce aspetti dell'opinione pubblica di notevole rilievo, anche se in seguito ampiamente offuscati dalla tradizione storiografica filosenatoria, ostile a Commodo soprattutto per effetto del contrasto che lo aveva opposto al senato.

Già riguardo ad uno dei primi atti del regno di Commodo, la pace conclusa nel 180 con i barbari, le fonti consentono, a mio avviso, di delineare l'esistenza di una tradizione contrapposta a quella ostile. Erodiano (I 6, 8-9), afferma che Commodo, corrotto dalle pressioni dei suoi servi e desideroso di ritornare al

${ }^{3} \mathrm{Si}$ è pensato, in particolare, che Draconzio abbia confusso Commodo con il padre Marco Aurelio (F. Vollmer, MGH, Auct. Ant., XIV, Berolini 1905, pp. 124, 298 e 441; P. Langlois, «Dracontius», Reallexicon für Antike und Christentum, IV, Stuttgart 1959, col. 261; M. St. Margaret, Dracontii Satisfactio, with Introd., Text, Transl. and Comm., Philadelphia 1936, p. 86; Draconzio. Satisfactio, Introduzione, testo, traduzione e commento di S. Gennaro, Catalia 1959, p. 56, n. 188) o con il poeta Commodiano (D. Kuijper, Varia Dracontiana, L'Aja 1958, pp. 52-3) o ancora con l'imperatore Lucio Vero (S. Mazzarino, «La Historia Augusta e la EKG», in Atti del Colloquio Patavino sulla Historia Augusta, Roma 1963, pp. 34-7; Id., Il pensiero storico classico, II 2, Bari 1966, 245-47 e, con qualche dubbio, C. Moussy, Dracontius. Oeuvres, II, Paris 1988, p. 213).

${ }_{4}^{4}$ Cf. in partic. D. Romano, Studi draconziani, Palermo 1959, pp. 51-2 e n. 138.

5 L. Alfonsi, "Commodo in Draconzio», RFIC, N.S. 39, 1961, pp. 296-300; Grosso, op. cit., p. 677 e n. 4; F. M. Clover, «Commodus the Poet», Nottingham Medieval Studies 32, 1988, pp. 19-33 (in partic. p. 20); B. Baldwin, «Commodus the good poet and good emperor: explaining the inexplicable», Gymnasium 97, 1990, pp. 224-231.

${ }^{6}$ In particolare, le lodi rivolte a Marco Aurelio e a Commodo da Atenagora $(\mathrm{Apol}$. 1, 24 e 37) non mi sembrano significative, poiché anche la tradizione ostile non nega le buone qualità dimostrate da Commodo durante il regno congiunto con il padre e subito dopo la morte di quest'ultimo, cercando anzi di spiegare il netto mutamento della condotta di Commodo con la nefasta influenza di cortigiani corrotti (Dio Cass., LXXIII 1, 1; Herodian., I $6,1-2 ; 6,8$ ). Il riferimento proprio a Commodo (cf. Alfonso, art. cit., p. 297; Grosso, loc. cit.) dell'elogio di Lattanzio (De mort. pers. 3, 4, Sources chrét., N. ${ }^{\circ}$ 39, Paris 1954, I, p. 82) dei multi boni principes che ressero l'impero fra Domiziano e Decio, permettendo il fiorire della Chiesa, mi sembra incerto, dato il carattere generico della testimonianza e l'esistenza in quel periodo di altri imperatori noti alla tradizione per il loro atteggiamento favorevole ai cristiani, in particolare Alessandro Severo e Felippo l'Arabo. 
più presto agli agi della vita a Roma, affidò il compito di difendere le rive del Danubio e respingere i barbari a persone di sua fiducia, che ben presto sottomisero la maggior parte dei barbari e convinsero gli altri a concludere un trattato che impegnava l'impero a pagar loro forti tributi ${ }^{7}$. Anche Cassio Dione condanna la pace come effetto del desiderio di Commodo di tornare a Roma $\mathrm{e}$, pur riferendo in maniera ben più obiettiva le condizioni imposte ai barbari, osserva che in tal modo Commodo perse l'occasionne di annientare i Marcomanni, che erano in gravi difficoltà ${ }^{8}$. Ancor più netta è poi la condanna del biografo dell'Historia Augusta, che accusa Commodo di aver abbandonato la guerra, accettando le condizioni imposte dai barbari ${ }^{9}$.

La pace del 180 è stata variamente giudicata dagli studiosi moderni, in relazione da un lato all'abbandono dei piani espansionistici che le fonti attribuiscono a Marco Aurelio, dall'altro alla sicurezza che essa poteva garantire ai confini dell'impero, e non sono mancate aspre critiche alla condotta di Commodo ${ }^{10}$. Tuttavia, al di là di queste valutazioni, basate su considerazioni strategiche e su una visione ex eventu dei fatti, ben più importante per il nostro assunto resta definire quali siano state le circostanze in cui fu conclusa la pace e quali le reazioni che essa provocò nell'opinione pubblica contemporanea. In proposito, si è sostenuto in particolare che la pace avrebbe costituito un vero e proprio tradimento ai danni dell'esercito, che della lunga guerra aveva sostenuto il peso ${ }^{11}$. Questa conclusione mi sembra tuttavia ben poco aderente all'effettiva reazione dei militari. In primo luogo, infatti, l'affermazione di Erodiano, secondo cui Commodo avrebbe fatto condurre le operazioni militari contro i barbari da persone di sua fiducia, è apertamente contraddetta da fonti pagane, Eurtropio e Aurelio Vittore, che testimoniano che la pace fu invece preceduta da vittorie ottenute personalmente da Commodo, del quale lodano

7 Quest'ultima affermazione è confermata, in tutt'altro contesto, da Cassio Dione (LXXIV 6, 1-2).

${ }^{8}$ Dio Cass, LXXIII 1, 2; 2, $1-4$.

${ }^{9} \mathrm{HA}$, Comm. 3, 5: bellum etiam, quod pater paene confecerat, legibus hostium addictus remisit ac Romam reuersus est.

${ }^{10} \mathrm{Cf}$. in particolare, con differenti valutazioni e con bibliografia, Grosso, op. cit., pp. 99101 e 457-67; G. Alföldy, «Die Friedenschluss Kaisers Commodus mit der Germanen», Historia 20, 1971, pp. 84-109 (rist. con Nachträge in: Id., Die Krise des römischen Reiches. Geschichte, Geschichtsschreibung und Geschichtsbetrachtung. Ausgewahlte Beiträge, Stuttgart 1989, pp. 25-68); M. Ššel Kos, A Historical Outline of the Region between Aquileia, the Adriatic, and Sirmium in Cassius Dio and Herodian, Ljubljana 1986, pp. 324-34; M. Stahl, «Zwischen Abgrenzung und Integration: die Verträge der Kaiser Mark Aurel und Commodus mit den Volkern jenseits der Donau», Chrion 19, 1989, pp. 289-317.

1) Grosso, op. cit., p. 102: «La chiusura completa delle operazioni... si risolse, dunque, ad ogni effetto, in un cattivo servizio reso, senza alcuna contropartita, ai comandanti e all'esercito, che vi avevano impegnato con le loro energie anche il loro orgoglio militare». 
le virtù belliche ${ }^{12}$; questa versione è ben nota pure alla tradizione cristiana ${ }^{13}$. Se, dunque, la pace fu preceduta da significativi successi, è evidente che Commodo poteva presentarla come frutto della vittoria; tale fu, in effetti, l'intento che egli perseguì per tutto il corso del suo regno, del quale egli volle appunto esaltare l'aspetto pacifico ${ }^{14}$. L'efficacia di tale ideologia dev'essere valutata tenendo presenti non le conseguenze della condotta di Commodo, bensì la situazione a lui contemporanea, caratterizzata soprattutto dalla profonda stanchezza diffusa per le lunghe guerre che avevano contraddistinto tutto il regno di Marco Aurelio.

In particolare, Cassio Dione (LXXII 15,6) attesta che Commodo, assunte il

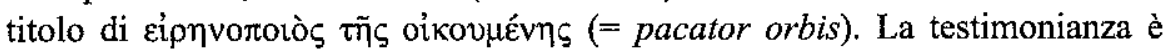
confermata da iscrizioni e papiri ${ }^{15}$ ed in particolare da un'iscrizione di Dura Europo, alla frontiera dell'Eufrate, posta su un altare dedicato al Genio di Dura da un decurione di cavalleria il 17 marzo del 193, in occasione dell'anniversario dell'ascesa al trono di Commodo, la cui morte, avvenuta il $1 .^{\circ}$ gennaio di quell'anno, doveva essere ancora ignota a Dura: Pro salu/te Com(modi)/ Aug(usti) Pii F(elicis)/ et uicto/riam d(omini) n(ostri)/ imp(eratoris), Pac(atoris)/ Orb(is), Inuict(i)/Rom(ani) Her[c(ulis) ${ }^{16}$.

L'iscrizione è di notevole interesse, sia per la sua collocazione cronologica alla fine del regno di Commodo, sia per la condizione di militare del dedicatario, che vale quindi a presentarla come una testimonianza significativa dell'atteggiamento dell'esercito; tanto più rilevante appare dunque, in questa

12 Eutrop. VIII 15, 1: contra Germanos feliciter et ipse pugnauit; Aur. Vict. Caes. 17, 2: bello plane impiger; quo in Quados prospere gesto, Septembrem mensem Commodum appellauerat (sull'erroneità di quest'ultima affermazione cf. Grosso, op. cit., pp. 460-61); cf. in particolare Alföldy, art. cit., p. 104.

13 Hieron., Chron., p. 208, 17 Helm: Commodus de Germanis triumphauit; Oros., VII 16, 2: aduersus Germanos bellum feliciter gessit; Iordan., Rom. 273: magnum triumphum de gente reuexit Germanica.

14 Per le attestazioni numismatiche di questo intento perseguito da Commodo fin dal 181 cf. in partic. J.-P. Martin, Prouindentia deorum. Aspects religieux du pouvoir romain, Rome 1982, pp. 344-46.

${ }^{15}$ CIL XIV 3449 (=ILS 400); $A E 1977,772$ (dove però il titolo è frutto d'integrazione). Per i papiri cf. P. J. Sijpesteijn, "Commodus' Titulature in Cassius Dio LXXII. 15. 5», Mnemosyne 41, 1988, pp. 123-24. Cf. P. Kneiss1, Die Siegestitulatur der römischen Kaiser,

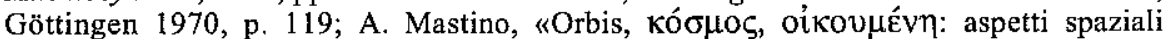
dell'idea di impero universale da Augusto a Teodosio», in Atti del III Seminario Internazionale di Studi storici «Da Roma alla terza Roma» (21-23 aprile 1983), Napoli 1984, pp. 85-6 (anche riguardo all'iscrizione faIsa $C I L, \mathrm{~V} 137$ ). In generale, su questo titolo, L. Berlinger, Beiträge zur inoffiziellen Titulatur der römischen Kaiser, diss. Breslau 1935 , pp. 47 ss. e la domentazione in Mastino, art. cit., pp. 136-37. Commodo fu il primo ad assumerlo.

${ }_{16}$ M. P. Speidel, "Commodus the God-Emperor and the Army», JRS 83, 1993, pp. 10914 , con nuove letture e precisazioni riguardo alla datazione. 
prospettiva, la scelta degli appellativi di Commodo, da Pacator Orbis a Pius e Felix, entrambi appellativi di pace, distinti dai cognomina ex uirtute ${ }^{17}$, ad Invictus ${ }^{18}$, come pure la dedica alla uictoria dell'imperatore. L'iscrizione conferma dunque che l'esaltazione delle qualità militari di Commodo, dimostrate nella campagna contro i barbari, e quella del regno nel suo complesso, come periodo di pace universale, sancita appunto dalle vittorie iniziali dell'imperatore, erano accettate anche dall'elemento militare, che dunque non si sentiva affatto tradito dalla pace conclusa nel 180 . Del resto, anche le fonti ostili, pur sforzandosi di universalizzare l'ostilità contro l'imperatore, devono riconoscere che, ancor dopo la sua morte, l'affetto dell'esercito verso Commodo si mantenne intatto ${ }^{19}$.

Un'ulteriore testimonianza di grande importand, generalmente trascurata ${ }^{20}$, è offerta poi da Eusebio. Questi, esponendo lo svolgimento della polemica sull'eresia montanista all'epoca appunto di Commodo, riferisce che uno scrittore anonimo, per confutare le profezie di Massimilla, seguace di Montano, la quale vaticinava l'avvento di un periodo di guerre e di disordini, aveva fatto

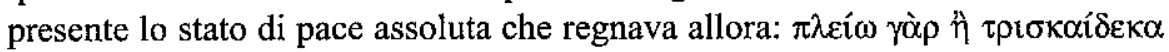

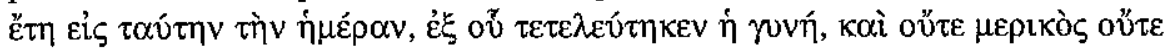

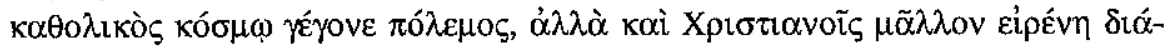

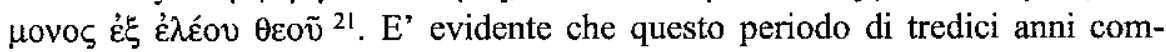
prendeva l'intero regno di Commodo e che l'anonimo antimontanista scriveva all'inizio del 192, prima dello scoppio delle guerre civili fra Settimio Severo e i suoi rivali. L'esaltazione del regno di Commodo come periodo di pace per i cristiani è comune, del resto, anche ad altre fonti, che sottolineano espressamente l'assenza di persecuzioni ${ }^{22}$, e risulta ben comprensibile, ove si consi-

17 Cf. Mazzarino, L'impero romano, II, rist. Bari 1986, p. 433. Commodo fu il primo a portare congiuntamente gli appellativi Pius e Felix: cf. C. Weinberger, «Felix», in: Diz. Epigr., III, Roma 1922, pp. 44-5; M. Hammond, "Imperial Elements in the Formula of the Roman Emperors during the First Two and a Half Centuries of the Empire», Mem. Amer. Acad. Rome 25, 1957, 33 ss.; A. Raubitschek, "Commodus and Athens», in Commemorative Studies in Honor of Th. L. Shear, Hesperia, Suppl. 8, 1949, p. 285; Martin, op. cit., p. $348^{\prime}$ ss.; E. Van't Dack, "Commode et ses épithètes Pius Felix sous les Sévères», in Historiae Augustae Colloquium Parisinum, a cura di G. Bonamente e N. Duval, Macerata 1991, pp. 311-35.

18 Dio Cass., LXXIII 15, 5, la cui testimonianza è ancora confermata da ILS 400 e dai papiri (Sijpesteijn, loc. cit).

19 Cf. ad es. Herodian. II 5, 7; HA, Pert. 6, 3.

${ }^{20}$ Su di essa ha richiamato giustamente l'attenzione S. Mazzarino, (L'impero romano, II, p. 433; cf. anche Id., Il basso impero. Antico, tardoantico ed èra costantiniana, I, Bari 1974 , p. 67); ma essa è stata del tutto trascurata in relazione all'analisi dei versi di Draconzio.

21 Euseb., Hist. eccl. V 16, 19. Su questa profezia cf. ad es. W. H. C. Frend, Martyrdom and Persecution in the Early Church, Oxford 1965, pp. 291-92.

22 Euseb., Hist. eccl. V 21, 1; Chron. Pasc., I, p. 489, 17-18 Dindorf. 
derino da un lato la politica tollerante di Commodo nei confronti dei cristiani ${ }^{23}$, dall'altro l'influenza su di lui della concubina Marcia, la quale indusse Commodo a liberare i cristiani detenuti in Sardegna, fra i quali il futuro papa Callisto ${ }^{24}$.

Ma la testimonianza dell'anonimo antimontanista va ben al di là dell'ambito limitato dei rapporti fra i cristiani e l'impero; egli, infatti, afferma che tutto il regno di Commodo, del quale non fa neanche il nome, sarebbe stato caratterizzato dall'assenza di guerre, sia particolari che generali, e proprio sull'affermazione di questo carattere pacifico dell'intero periodo, considerato come un dato di fatto generalmente ammesso e incontrovertibile, basa la propria confutazione delle profezie di Massimilla. Questa universalità e, insieme, il carattere incidentale della testimonianza valgono, a mio avviso, ad attribuirle un valore peculiare, come espressione di un giudizio favorevole non limitato all'ambito cristiano e non motivato solo dalla condotta di Commodo in campo religioso; essa conferma, dunque, che la propaganda di Commodo riguardo all'aspetto pacifico del suo regno aveva prodotto profondi effetti, in relazione anche con il diffuso sentimento di stanchezza, dopo le lunghe guerre che avevano caratterizzato il regno di Marco Aurelio.

Un'analoga visione del regno di Commodo come periodo di pace, caratterizzato da opere civili e di ricostruzione, è sottintesa nell'ampia narrazione offerta dal cristiano Giovanni Malala, cronista antiocheno del vI d.C. ${ }^{25}$. Questi

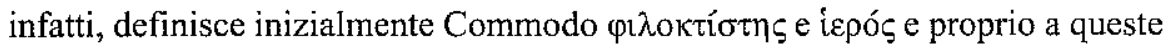
due caratteristiche dell'imperatore, l'amore per le costruzioni e l'interesse per i culti, è dedicata l'intera narrazione di Malala, che enumera la costruzione di bagni pubblici detti Commodiani, di un tempio di Atena, del Sisto e di un tempio a Giove Olimpio ${ }^{26}$ e si dilunga poi sulla nuova organizzazione data

23 Cf. in partic. Grosso, op. cit., pp. 669-78; M. Sordi, Il cristianesimo e Roma, Bologna 1965, pp. 199-207; P. Keresztes, «A Favourable Aspect of the Emperor Commodus' Rule», in Hommages à M. Renard, II, 'Coll. Latomus' 102, Bruxelles 1969, pp. 368-77.

${ }_{24}$ Ippol., Refut. IX 12 (P. Wendland, Hippolytus Werke, III, Leipzig 1916, pp. 246-48). Sulla vicenda cf. in partic. Grosso, op. cit., pp. 670 ss.; Sordi, op. cit., pp. 203-6; Mazzarino, Il basso impero, I, pp. 54 ss.; Id., L'impero romano, II, pp. 451 ss.

${ }^{25}$ Su di lui cf. in partic. G. Moravcsiik, Byzantinoturcica, I, Berlin 1958, pp. 329-34; G. Downey, A History of Antioch in Syria from Seleucus to the Arab Conquest, Princeton, New Jersey 1961, pp. 38-40; A. Momigliano, «Malalas», in The Oxford Classical Dictionary, Oxford $1970^{2}$, p. 641; H. Hunger, Die Hochspracliche Profane Literatur der Byzantiner, I, München 1978, pp. 319-26; B. Croke, in: AA.VV., Studies in John Malalas, ed. by E. Jeffreys, with B. Croke and R. Scott, Sidney 1990, pp. 1-26 con amplia bibliografia. Per il testo si veda l'edizione di A. Schenk von Stauffenberg (Die römische Kaisergeschichte bei Malalas, Stuttgart 1931); preferisco comunque citarlo in base alle pagine di quella di L. Dindorf (Bonnae 1831), perché più generalmente nota e pủ facilmente reperibile.

26 Malal., Chron., p. 283; cf. von Stauffenberg, op. cit., pp. 452-53; Downey, op. cit., p. 233; Grosso, op. cit., pp. 82-3 e 575-76. 
allora ai giochi olimpici di Antiochia, sui finanziamenti concessi dall'imperatore per il loro svolgimento, sull'autorizzazione ad usare per essi il lascito di Sosibio e sullo svolgimento dei giochi ${ }^{27}$, ricordando anche che gli Antiocheni eressero a Commodo una statua di bronzo nel centro della città ${ }^{28}$. Malala attesta infine che, in occasione del terremoto che distrusse Nicomedia, Commodo offrì grandi munificenze e ricostruì la città ${ }^{29}$.

Questa descrizione elogiativa del regno di Commodo non è dovuta, a mio avviso, all'influenza della tradizione cristiana. Il racconto di Malala deriva infatti da fonti locali antiochene di epoca imperiale, in particolare $i$ cronisti Pausania e Domnino ${ }^{30}$; inoltre, 1'interesse di Commodo per il rispristino di feste e giochi, le sue munificenze e le costruzioni di edifici sacri si riferiscono a culti e cerimonie pagane, senza il benché minimo accenno al cristianesimo. Mi sembra dunque chiaro che, nonostante l'adesione di Malala al cristianesimo, la sua narrazione del regno di Commodo è animata soprattutto da un particolare interesse per le antichità, i riti e gli edifici di culto pagani, avvertibile del resto in tutta la sua opera ${ }^{31}$ e dovuta all'influenza di fonti pagane, più vicine di lui all'epoca di Commodo e che riflettevano la soddisfazione che l'operato di questo imperatore aveva suscitato ad Antiochia.

Questo atteggiamento appare pienamente comprensibile, se teniamo presente che Marco Aurelio, per punire gli Antiocheni di aver parteggiato per Avidio Cassio, aveva vietato loro per qualche tempo di organizzare spettacoli e di tenere qualsiasi riunione pubblica e li aveva privati di alcuni privilegi ${ }^{32}$; le

${ }^{27}$ Malal., Chron., pp. 284-89; cf, von Stauffenberg, op. cit., pp. 412 ss.; Downey, op. cit., pp. 232-35; L. Robert, «Epigraphica XI», REG 49, 1936, pp. 11-2 (= Opera minora selecta, II, Amsterdam 1969, pp. 949-59); Grosso, op. cit., pp. 83-4 e 575-76.

28 Malal., Chron., p. 286, 4-5; cf. von Stauffenberg, op. cit., p. 453; Downey, op. cit.. p. 234, n. 161. Inoltre, l'istituzione di una festa in onore di Commodo è attestata da iscrizioni (Downey, op. cit., p. 235 e n. 162).

29 Malal., Chron., p. 289, 11-2.

${ }^{30} \mathrm{Cf}$. H. Bourier, Über die Quellen der ersten vierzehn Bücher des Johamnes Malalas, 1-II, Progr. Ausburg 1899; von Stauffenberg, op. cit., pp. 81 ss. e 327 ss.; Grosso, op. cit., pp. 83 e 85 ; E. Jeffreys, in: AA.VV., Studies... cit., pp. 168 ss. Non mi sembra convincente l'ipotesi del Grosso (op. cit., pp. 86 e 552), secondo cui l'accenno al terremoto di Nicomedia deriverebbe da Cassio Dione. Non solo, infatti, è ben nota la tendenza di Malala ad ignorare la narrazione degli storici più accreditati per seguire quella dei cronisti locali, ma anche l'accenno di Cassio Dione (LXXIII 12,2) ai benefici che Nicomedia potè ottenere grazie ai buoni uffici del suo potente cittadino Saotero, se si riferisce, come intende if Grosso, appunto a munificenze conseguenti il terremoto, non trova alcuna corrispondenza nel racconto di Malala, in cui invece è messa in forte rilievo l'iniziativa personale di Commodo riguardo alle liberalità verso Nicomedia.

$3 \mathrm{Cf}$. in partic. Downey, «Imperial Building Records in Malalas», B!:- Zeitschr. 38, 1938, pp. 1-16 e 299-311; A. Moffatt, in: AA.VV., Studies..., pp. 87-109.

32 HA, M. Ant. 25, 8-9; Av. Cass. 9, 1; cf. Downey, A Histor $3 \ldots$... p. 228. Mi sembra da sottolineare, in proposito, il contrasto fra la lunga narrazione che Malala dedica a Commodo 
iniziative di Commodo per il ripristino, l'ampliamento e la sovvenzione degli spettacoli non potevano dunque che suscitare l'entusiasmo e degli Antiocheni e far sì che il so regno restasse nella memoria locale come un'epoca felice, compresa com'era fra le vicende del tempo di Marco Aurelio e quelle, di poco successive, del regno di Settimio Severo, che punì anch'egli la città per aver parteggiato per Pescennio Nigro ${ }^{33}$. La versione locale seguita da Malala mi sembra dunque testimonianza di una tradizione favorevole a Commodo che potè essere abbastanza diffusa nelle province, per effetto del periodo di pace e dei benefici ricevuti; né certo diverso sarà stato il giudizio degli abitanti di Nicomedia, la cui città, ricostruita dall'imperatore, aveva anche ottenuto l'istituzione di un agone e di un tempio in onore di Commodo e la concessione della seconda neocoria, che in seguito le venne tolta ${ }^{34}$. Le iniziative di altre città dell'Oriente, che aggiunsero il nome di Commodo alle denominazioni di feste già esistenti, attestano infine analoga riconoscenza per benefici ricevuti ${ }^{35}$.

L'importanza di queste benemerenze per la formazione ed il persistere di un giudizio favorevole su Commodo in ambito provinciale mi sembra del resto sottolineata soprattutto dal fatto che l'elogio di Malala del iepós Commodo e l'enumerazione degli edifici da lui fatti costruire ad Antiochia si contrappongono nettamente alle feroci critiche che la tradizione filosenatoria rivolse all'imperatore proprio riguardo a questo aspetto della sua opera. Infatti, il biografo dell'Historia Augusta condanna aspramente la povertà dell'opera monumentale di Commodo, affermando che l'unico edificio rimasto, le terme Commodiane, sarebbe stato costruito a suo nome dal ministro Cleandro e che Commodo avrebbe posto il suo nome su opere non sue e non avrebbe neanche portato a termine le opere iniziate dal padre ${ }^{36}$; mentre Aurelio Vittore esprime un giudizio assai aspro perfino sulle terme, che considera indegne della potenza di Roma ${ }^{37}$. L'elogio di Malala dell'attività edificatoria di Commodo ad

e quella brevissima (Chron., pp. 281, 20-282, 13) in cui condensa il regno di Marco Aurelio.

${ }^{33}$ Herodian., III 6, 9; HA, Seu. 9, 4; Dig., L 15, 1, 3; Downey, A History..., p. 240.

34 Dio Cass., LXXIII 12, 2; B. Pick, «Die tempeltragenden Gottheiten und die Darstellung der Neokoris auf den Münzen», Jahresh. öst. arch. Inst. 7, 1904, p. 7, III, nrr. 1-2 e p. 26; S. R. F. Price, Rituals and Power. The Roman Imperial Cult in Asia Minor, Cambridge 1984 , p. 266.

${ }_{35}$ Cf. D. Magie, Roman Rule in Asia Minor to the End of the Third Century after Christ, Princeton, New Jersey 1950, I, p. 668; II, p. 1538; T. T. Duke, «The Festival Chronology of Laodicea ad Lycum», in Studies Presented to D. M. Robinson, II, Saint Louis 1953, pp. 853-54. Per altri onori resi a Commodo cf. ad es. Price, op. cit., pp. 85, 118 e 250.

$36 \mathrm{HA}$, Comm. 17, 5-7: opera eius praeter lauacrum, quod Cleander nomine ipsius fecerat, nulla extant. Sed nomen eius alienis operibus incisum senatus erasit. Nec patris autem sui opera perfecit.

${ }^{37}$ Aur. Vict., Caes. 17, 3: moenia Romae potentia uix digna lauandi usui instituit. 
Antiochia sembra dunque quasi controbattere le critiche della tradizione ostile ed è comunque indicativo di un giudizio del tutto opposto in ambito provinciale. La questione non mi sembra affatto secondaria, se si tiene presente che la costruzione di edifici pubblici, oltre ad avere importanti riflessi per la vita economica, era considerata compito precipuo dell'imperatore e tale da condizionare il giudizio complessivo sulla sua attività ${ }^{38}$.

Conviene a questo punto ricordare che il regno di Commodo fu caratterizzato in Africa da una notevole prosperità ${ }^{39}$, accresciuta dal particolare interesse dell'imperatore per i rifornimenti granari provenienti da essa ${ }^{40}$; furono inoltre realizzati importanti lavori per il rafforzamento del limes, per il miglioramento della rete stradale ${ }^{41}$ e per la costruzione e il restauro di templi e di opere pubbliche ${ }^{42}$, la municipalizzazione ebbe ulteriore sviluppo ${ }^{43}$ e l'intervento diretto dell'imperatore, attestato in particolare nel caso dei coloni del saltus Burunitanus ${ }^{44}$, migliorò la situazione sociale e suscitò speranze in uno sviluppo ancor maggiore ${ }^{45}$. La concessione a Cartagine del titolo di Alexandria Commodiana togata ${ }^{46}$ conferma del resto il particolare favore dell'imperatore verso quella città e l'Africa fu la meta dell'unico viaggio progettato, anche se non effettuato, de Commodo durante il suo regno ${ }^{47}$. Infine, anche in Africa il regno di Commodo sembra essere stato caratterizzato da tolleranza verso i cristiani ${ }^{48}$. Tenendo presenti tutti questi elementi, sembra logico ritenere che il regno di Commodo avesse lasciato in Africa un ricordo sostanzial-

38 Per queste concezioni, che nelle province orientali trovavano una base nell'evergetismo dei sovrani ellenistici, basti qui rimandare alle considerazioni generali di P. Veyne (Il pane $e$ il circo, trad. it., Bologna 1984, pp. 559 ss.).

${ }^{39} \mathrm{Cf}$. in partic. P. Romanelli, Storia delle province romane dell'Africa, Roma 1959, pp. 380 ss.; Grosso, op. cit., pp. 618 ss.

40 Cf. Marasco, Studia Historica, Firenze 1988, pp. 51-57.

41 Cf. ad es. Romanelli, op. cit., pp. 383-85; Grosso, op. cit., pp. 616-18.

42 Cf. Romanelli, op. cit., pp. 389-91; Grosso, op. cit., pp. 621 ss.

${ }^{43}$ Cf. Romanelli, op. cit., pp. 388-89; Grosso, op. cit., pp. 621-22; J. Gascou, La politique municipale de l'Empire romain en Afrique Proconsulaire de Trajan à Septime-Sévère, Rome 1972, pp. 162-67 e 222-26.

44 FIRA I $103=$ P. F. Girard - F. Senn, Les lois des Romains, 7.e édition par un groupe de romanistes, Camerino 1977 , pp. $68-71=$ D. P. Kehoe, The Economics of Agriculture on Roman Imperial Estates in North Africa, Göttingen 1988, p. 56.

${ }^{45}$ Cf. Grosso, op. cit., p. 621, sulla base di CIL, VIII 23828.

46 HA, Comm. 17, 8.

47 HA, Comm. 9, 1.

48 Tertulliano (Ad Scap. 4, 3) ricorda, ad es., la liberazionne di un cristiano da parte di Vespronio Candido proconsole sotto Commodo (cf. Romanelli, op. cit., p. 381; Grosso, op. cit., pp. 595 e 677). La vicenda dei martiri di Scilli, condannati a Cartagine il 17 luglio del 180 (cf. Atti e passioni dei martiri, a cura di A. A. R. Bastiaensen et al., Milano 1987, pp. 97-105 e 405-11, con bibliografia), non è affatto indicativa, poiché a quella data Commodo era appena asceso al trono e il processo non fu certo giudicato in base alle sue direttive (cf. ad es. Grosso, op. cit., p. 669). 
mente positivo, ancor vivo nella tradizione locale al tempo di Draconzio ${ }^{49} \mathrm{e}$ tale da influire, insieme alla tradizione cristiana, sul giudizio che egli espresse su Commodo.

Il quadro delle testimonianze che abbiamo esaminate aiuta dunque a comprendere la genesi della tradizione favorevole a Commodo, alla quale si ricollega Draconzio; né all'attribuzione dei versi draconziani a Commodo si oppone il silenzio delle fonti su un'attività poetica di questo imperatore ${ }^{50}$. Infatti, anche se la tradizione ostile si compiace di raffigurare Commodo dedito ai piaceri e ai bagordi, è ben attestata l'ottima educazione letteraria che egli ricevette in gioventù, che fu cura precipua del padre. Lo stesso Marco Aurelio ringraziava gli dèi d'aver trovato ottimi maestri per i suoi figli ${ }^{51}$ ed Erodiano (I 2,1$)$ attesta che egli aveva chiamato da tutte le province i maestri più famosi perché educassero Commodo. L'Historia Augusta (Comm. 1, 5-6), infine, testimonia che Marco cercò di educare Commodo... et suis praeceptis et magnorum atque optimorum uirorum e riferisce i nomi di alcuni di questi insegnanti: il maestro di greco Onesicrate, quello di latino Antistio Capella, non altrimenti attestati, e quello di retorica, T. Aio Santo ${ }^{52}$, che è invece noto per gli importanti incarichi che rivestì nell'amministrazione ${ }^{53}$. Commodo ricevette dunque una buona educazione letteraria e non stupisce affatto che abbia potuto comporre versi come quelli riferiti da Draconzio ${ }^{54}$, il cui contenuto, sostanzialmente banale, riflette, del resto, la filosofia e lo stile di vita del padre Marco Aurelio.

\section{Gabriele Marasco}

49 Mi sembra interessante sottolineare, in questa prospettiva, la consonanza della pietas per cui Draconzio loda Commodo con l'analogo attributo ípóç in Malala (Chron, p. 283, 4), che segue la notazione dell'amore di Commodo per le costruzioni e si riferisce con ogni evidenza alla sua cura per le cerimonie e per gli edifici di culto: è a mio avviso credibile che l'espressione draconziana si ricolleghi a un'analoga tradizione elogiativa della condotta di Commodo verso i culti locali.

50 Difficoltà notata, ad es., dal Mazzarino (cit. supra, nota 3).

51 Medit. 117.

52 Nei mss. ricorre la forma Ateius Sanctus, ma il nome esatto è attestato da un' iscrizione (L. Moretti, «Due iscrizioni latine inedite di Roma», RFIC, N.S. 38, 1960, pp. 68-73=AE 1961, 280).

${ }_{53}$ Cf. Moretti, loc. cit.; H.-G. Pflaum, Les carrières procuratoriennes équestres sous le Haut-Empire romain (Supplément), Paris 1982, pp. 109-10; A. Birley, Marco Aurelio, trad. it., Milano 1990 , p. 248 . E' ancora da ricordare il particolare favore dimostrato da Commodo già imperatore nei riguardi di alcuni sofisti (Philostr., V. soph. 590; 593).

54 Riguardo al significato dell'espressione modico sermone poeta, l'interpretazione dell'Alfonsi (art. cit., p. 399), «poeta di brevi composizioni, di carmi esigui, di massime concise», mi sembra da preferire a quelle che intendono l'espressione come un giudizio negativo sulle qualità poetiche di Commodo (così ancora Moussy, op. cit., II, p. 185: «poète au style peu chatiés). Sulle occasioni in cui Commodo può aver composto i versi riferiti da Draconzio cf. Baldwin, art. cit., pp. 228-29. 